\title{
Monte Carlo study of the relation between vacancy diffusion and domain growth in two-dimensional binary alloys
}

\author{
Carlos Frontera, Eduard Vives, and Antoni Planes \\ Departament d'Estructura i Constituents de la Matèria, Universitat de Barcelona, \\ Diagonal 647, Facultat de Física, E-08028 Barcelona, Catalonia, Spain
}

(Received 15 March 1993; revised manuscript received 21 June 1993)

\begin{abstract}
Domain growth in a two-dimensional binary alloy is studied by means of Monte Carlo simulation of an $A B V$ model. The dynamics consists of exchanges of particles with a small concentration of vacancies. The influence of changing the vacancy concentration and finite size effects has been analyzed. Features of the vacancy diffusion during domain growth are also studied. The anomalous character of the diffusion due to its correlation with local order is responsible for the obtained fast-growth behavior.
\end{abstract}

\section{INTRODUCTION}

The study of growth kinetics in systems rapidly quenched through a phase transition has been a subject of vivid interest during the past decade. ${ }^{1-3}$ Numerical studies of simple kinetic Ising models ${ }^{4}$ and Langevin models ${ }^{5}$ reveal that, in recent times, the nonequilibrium structure factor scales with the average of the inverse of the domain size $R(t)^{-1}$. In this regime, $R(t)$ grows with time according to a power law $R(t) \sim t^{x}$, where $x$ is the growth exponent and it is found to be quite universal: It depends on only a few parameters and mainly on whether the order parameter is conserved or not. This feature enables one to classify, in a limited number of classes, different systems exhibiting a wide variety of growth processes. Measurements on different experimental systems seem to corroborate the scaling and power-law growth behaviors. ${ }^{6-9}$ Nevertheless, the universality of the exponent is a more controversial point. Indeed, it is worth noticing that measurements are difficult to perform and in most cases even to interpret.

A representative problem of growth kinetics is the ordering process in an $A-B$ binary alloy quenched from an initial temperature above its order-disorder transition temperature $T_{c}$ down to a final temperature $T$, below $T_{c}$. In this case, immediately after the quench, the system is in a disordered state characteristic of the hightemperature phase. Due to fluctuations, the disordered state decays to the equilibrium state corresponding to the temperature $T$. For a given composition and shallow quenches $\left(T_{c}-T<<T_{c}\right)$ the evolution to equilibrium usually initiates through the nucleation and growth mechanism but for sufficiently deep quenches $\left(T<<T_{c}\right)$, the early evolution occurs through the spinodal ordering mechanism. In both cases, at late times, an interpenetrating array of domain walls separating ordered regions appears. The number of different ordered regions depends on the ground-state degeneracy. This is the coarsening or domain growth regime.

Usually, this problem has been directly mapped on the spin-exhange kinetic Ising model, ${ }^{10,11}$ interpreting $A$ atoms as spin up and $B$ atoms as spin down, and has been numerically simulated using the Monte Carlo technique. For this model, at late times, it is found that $R \sim t^{x}$ with $x=1 / 2$, which is the expected result for a system with a nonconserved order parameter (Allen-Cahn law). ${ }^{12}$

The spin-exchange dynamics supposes the direct interchange of neighboring $A$ and $B$ atoms. This mechanism is not realistic for the case of alloys, where it is known that interdiffusion proceeds through vacancies. ${ }^{13}$ Recently we have incorporated this vacancy mechanism to study ordering kinetics in a $2 d$ binary alloy in a square lattice undergoing an order-disorder transition. ${ }^{14,15}$ In this case only exhanges of $A$ or $B$ atoms with a vacancy are possible. If only nearest neighbors (NN) vacancy jumps are allowed, at late times, a logarithmic growth is obtained in agreement with results reported by Mouritsen and Shah. ${ }^{16,17}$ This behavior has been attributed to the trapping of vacancies in ordered regions. Nevertheless if a small fraction of next-nearest neighbors (NNN) jumps are permitted, a power-law behavior with an exponent $x$ greater than $1 / 2$ is found. It has also been shown that in the domain growth regime dynamical scaling holds. ${ }^{14}$ This anomalous fast behavior has been attributed to nonuniform excitations arising from the interaction of the system with a heat bath, which modify in a nonlinear way the time scale. ${ }^{15}$

In previous papers, ${ }^{14,15}$ the dynamics was introduced by a single vacancy on systems of different sizes. This made it difficult to separate the effect of vacancy concentration from finite-size effects. In this work, we separately analyze the influence of both effects on the dynamical exponent $x$. Moreover, the special features of the growth kinetics are related to the characteristics of vacancy diffusion. The results presented correspond to $2 d$ stoichiometric binary alloys in a square lattice at relatively low temperature. As the fast growth behavior is attributed to the nonhomogeneity of excitations, we expect that increasing temperature has an important effect on the growth kinetics. So, the effect of temperature has also been studied.

The paper is organized as follows: In Sec. II we in- 
troduce the model. In Sec. III we present the Monte Carlo simulation details. In Sec. IV we expose the results and, finally, in Sec. $\mathrm{V}$ we discuss the main points and conclude.

\section{MODEL}

We have considered the standard $A B V$ model for the description of an $A B$ binary alloy ${ }^{18,19}$ with vacancies and nearest-neighbors (NN) interaction. The system is defined on a $2 d$ square lattice with dimensions $L \times L$ and periodic boundary conditions. On each site we define a variable $S_{i}\left(i=1, \ldots, N=L^{2}\right)$ taking values $+1,-1$, or 0 if that site is occupied by an $A$ atom, $B$ atom, or a vacancy $(V)$, respectively. The particle and vacancy concentrations are given by $c_{A} \equiv N_{A} / N, c_{B} \equiv N_{B} / N$, and $c_{V} \equiv N_{V} / N$, where $N_{A}, N_{B}$, and $N_{V}$ are the number of particles of each kind, which are constant. We have focused on the stoichiometric case fulfilling $c_{A} \simeq c_{B} \simeq 0.5$ and $c_{V} \leq 4 \times 10^{-4}$. For such a low concentration of vacancies the $A B V$ model can be reduced to an Ising-like model, whose Hamiltonian reads

$$
H=J \sum_{i j}^{\mathrm{NN}} S_{i} S_{j}
$$

where the sum is performed over all the NN pairs, and $J$ is a positive constant in order to ensure the antiferromagneticlike ordering of the particles. The ground state consists of two alternating sublattices filled by $A$ and $B$ particles respectively. The vacancies are symmetrically distributed on the two sublattices, and have a tendency to cluster, since there is an effective energy difference $J$ between a separated and a NN-bounded pair of vacancies. Besides the high degeneracy due to the vacancy positions, there is an intrinsic degeneracy due to the possibility for two kinds of alternating $A B$ domains. This model exhibits an order-disorder phase transition at practically the same temperature as the $2 d$ Ising model $\left(T_{c} \simeq 2.26 \mathrm{~J} / k_{B}\right)$. Above this temperature, the system shows a completely disordered state in which the vacancies show no effective interaction.

\section{MONTE CARLO SIMULATION DETAILS}

Monte Carlo simulations have been performed using the standard Metropolis algorithm. The dynamics in the system is introduced by moving only the vacancies, which can perform jumps to NN and NNN positions. The existence of jumps to NNN positions has been shown to be crucial in order to prevent vacancy trapping phenomena. ${ }^{15}$ The ratio between the proposed jumps to the NN positions and the total number of proposed jumps (NN and NNN positions) is taken to be $W=0.5$, which means that the jumps to the eight neighboring positions are equally probable. The dependence of the results on this parameter has also been studied in a previous paper ${ }^{15}$ showing that only when $W$ is very close to 1 is the kinetics affected. In that case, a logarithmic growth is obtained at late times.
The system is quenched from a completely disordered state $(T=\infty)$ to a temperature $T$ below $T_{c}$ and its subsequent evolution is monitored as a function of time, which is measured in Monte Carlo step (MCS) units. A MCS consists of $N$ vacancy jump trials. Although the vacancies are selected sequentially in order to improve the simulation speed, no time correlations have been found due to this fact. Our definition of a MCS gives a time scale independent of the number of vacancies in the system.

Systems of linear sizes $L=50,100,200,500$, and 1000 with periodic boundary conditions have been simulated in order to study finite-size effects. We have also studied different vacancy concentrations, ranging from $c_{V}=10^{-6}$ to $c_{V}=4 \times 10^{-4}$.

The initial state of the system is generated by randomly placing an equal number of $A$ and $B$ atoms on the lattice and, after, uniformly picking up $N_{V}$ particles. Most of the simulations have been performed at $T^{*}=k_{B} T / J=1.0$, which is well below the critical temperature $T_{c}$.

The following quantities have been measured after each MC step.

(a) Excess energy per site $\Delta E=\left[E(t)-E_{\text {eq }}\right] / N$, where $E(t)$ is the total energy of the system, and $E_{\text {eq }}$ is the energy of the system when equilibrium is reached. This value has been measured after long time simulations $(\simeq$ $10^{5}$ MCS's) and within a relative accuracy of $10^{-4}$ shows no difference with the exact values for the $2 d$ Ising model.

(b) Order parameter $m=\left[\sum_{i} S_{i} \operatorname{sgn}(i)\right] / N$, where $\operatorname{sgn}(i)$ takes alternating values +1 and -1 over the square lattice in a chessboardlike way.

(c) Number of vacancy-vacancy exchanges per site $\Phi_{V V}$. This quantity is measured, in the case of having more than one vacancy on the system, in order to analyze if time corrections are needed due to the possible nonphysical significance of such exchanges.

(d) Effective vacancy diffusion constant $\tilde{D}(t)$, which is defined, for a system with a single vacancy, as

$$
\tilde{D}(t)=\frac{\sum_{k=1}^{N}\left(\mathbf{r}_{k}-\mathbf{r}_{0}\right)^{2}}{N^{2}}
$$

where $\mathbf{r}_{k}$ indicates the vacancy position at the $k$ th-jump proposition during the MC step and $\mathbf{r}_{0}$ indicates the initial vacancy position at the beginning of the $\mathrm{MC}$ step.

The quantities above are averaged over a large number of independent runs (around 75 runs for $L=50$ and 100, 35 runs for $L=200,20$ runs for $L=500$, and 15 runs for $L=1000$ ), starting with different random number generator seeds. These sets of runs do not include those that lead to final "slab" configurations corresponding to two competing domains separated by flat interfaces. Such slab runs represent $30 \%$ of the total number of runs performed. For the effective vacancy diffusion constant, the averages have been increased up to 400 independent runs for $L=100$ and 200 runs for $L=500$.

Simulations have been carried out on an IBM $3090 / 600 \mathrm{VF}$ computer and have taken around $200 \mathrm{CPU}$ hours. We have designed an improved algorithm which 
achieves a speed of 10 MCS's/min in a $1000 \times 1000$ system.

\section{RESULTS}

The existence of scaling in a broad temporal regime has been tested in a previous work by measuring the structure factor evolution. Figure 1 shows a typical loglog plot of the excess energy $\Delta E$ and of the order parameter $m$ versus time, corresponding to a system with $L=200$ and $N_{V}=16$. Three different regimes can be distinguished. First an initial transient regime (0-50 MCS's) in which ordered domains separated by domain walls are formed. Second a domain growth regime (501000 MCS's) in which scaling holds and the excess energy and the order parameter show a power-law dependence with time,

$$
\Delta E \sim t^{-x_{e}}, \quad m \sim t^{x_{\mathrm{op}}},
$$

and finally a finite-size effect regime in which the excess energy shows a very fast decrease and the equilibrium value $E_{\text {eq }}$ is reached. In the intermediate regime, if scaling holds and the excess energy only depends on the amount of interface present in the system, the exponents measured from the order-parameter evolution $x_{\mathrm{op}}$ and from the excess energy evolution $x_{e}$ must satisfy $x_{\mathrm{op}}=\frac{d}{2} x_{e}{ }^{20}$ where $d$ is the space dimensionality. We have checked such a relation for most of our simulations and found that it is always fulfilled within the errors of the fitted exponents. Since the statistical error associated to the order parameter evolution is greater than the error associated to the energy, we concentrate on the measurement of the exponent fitted from the excess en-

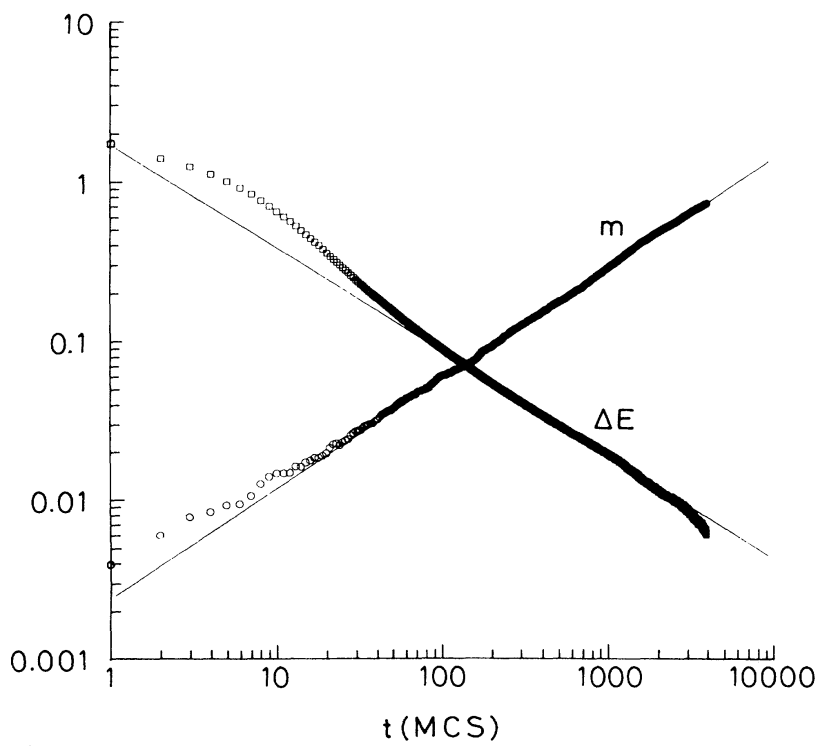

FIG. 1. Typical log-log plot of the excess energy $\Delta E$ and order parameter $m$ versus time for a system of size $L=200$ and $N_{V}=16$. Data correspond to an average over 33 different processes. The lines are linear square fits. ergy evolution. In the following we will refer to the $x_{e}$ exponent as the growth exponent $x$.

\section{A. Vacancy concentration dependence}

We have performed runs in systems with different numbers of vacancies in order to analyze the dependence of the growth exponent with the vacancy concentration. Figure 2 shows the excess-energy evolution in a log-log plot for a system with $L=500$ and vacancy concentrations $c_{V}=4 \times 10^{-4}, 10^{-4}, 2.5 \times 10^{-5}$, and $4 \times 10^{-6}$, together with the fitted linear behaviors. It can be seen that the slopes are nearly constant. Table I shows the fitted exponents corresponding to all the studied cases for different sizes and concentrations. The dependence of $x$ with the concentration of vacancies is very small. A little systematic decrease of $x$ for increasing $c_{V}$ can, however, be appreciated. Such decrease could be associated to the existence of vacancy-vacancy exchanges during the simulation whose physical meaning could be controversial. For this reason we have studied the statistics of the number of vacancy-vacancy exchanges $\Phi_{V V}$ during the simulation. In Fig. 3 we present the evolution of $\Phi_{V V}$ as a function of time. The data correspond to a system with $L=1000$ and $N_{V}=400$, averaged over 13 independent runs. The behavior of $\Phi_{V V}$ can be understood in terms of the formation of domain walls. At the beginning of the evolution, when the system is completely disordered, one has $\Phi_{V V} \simeq 4 \times 10^{-4}$, which is the expected value corresponding to the case in which there is no vacancyvacancy correlations. During the first $100 \mathrm{MC}$ steps an effective attraction between the vacancies appears due to

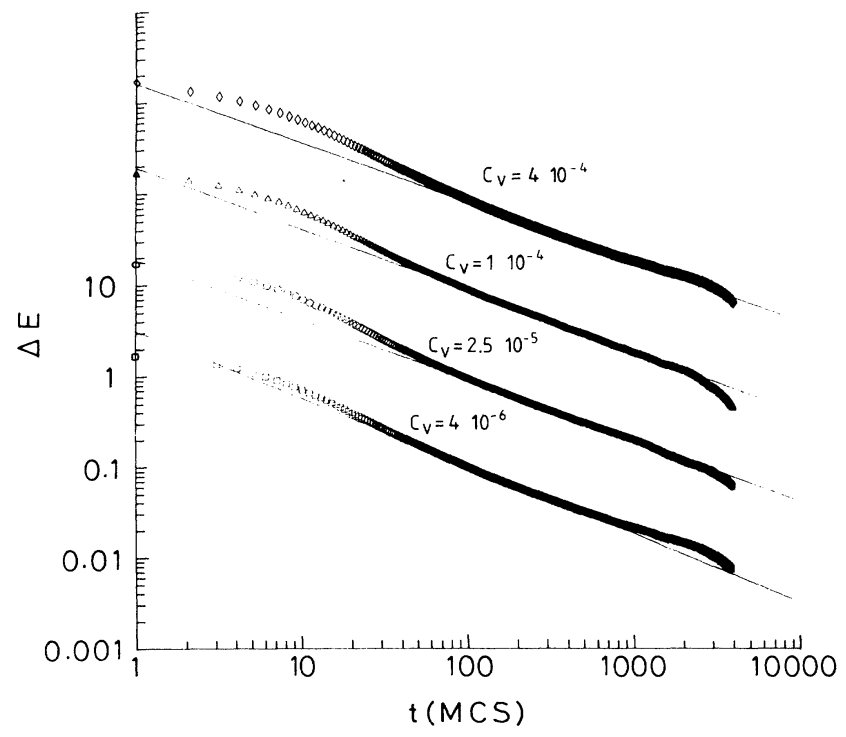

FIG. 2. Log-log plots of the evolution of the excess energy $\Delta E$ versus time for a system with size $L=500$ and different vacancy concentration $c_{V}$. Data correspond to an average over 20 different runs. The curves are shifted one decade in order to clarify the picture. The lines are linear square fits whose slope is nearly constant. 
TABLE I. Fitted growth exponents $x$ for systems with different sizes $L$ and different vacancy concentrations $c_{V}$.

\begin{tabular}{cccccc}
\hline \hline$c_{V}$ & $4 \times 10^{-4}$ & $10^{-5}$ & $2.5 \times 10^{-5}$ & $4 \times 10^{-6}$ & $10^{-6}$ \\
$L$ & & & & \\
\hline 50 & $0.98 \pm 0.02$ & & & \\
100 & $0.90 \pm 0.02$ & $0.91 \pm 0.02$ & & & \\
200 & $0.73 \pm 0.02$ & $0.76 \pm 0.02$ & $0.80 \pm 0.02$ & & \\
500 & $0.65 \pm 0.02$ & $0.67 \pm 0.04$ & $0.67 \pm 0.02$ & $0.75 \pm 0.03$ & \\
1000 & $0.67 \pm 0.02$ & & & & $0.69 \pm 0.03$ \\
\hline \hline
\end{tabular}

the combination of two effects: the formation of domain walls and the tendency of the vacancies to stay in the domain walls. The total length of domain walls has a maximum just before the domain growth regime starts. During this regime the number of domain walls decreases, which implies a decrease in $\Phi_{V V}$. Although the time evolution of $\Phi_{V V}$ is important, its absolute value, even at the maximum, is very small compared to the number of total proposed exchanges. We have checked that the possible corrections to the time scale due to this effect are irrelevant.

\section{B. Finite-size dependence}

Figure 4 shows five different evolutions of the excess energy decay corresponding to the same vacancy concentration $c_{V}=4 \times 10^{-4}$ and systems of size $L=50$, $100,200,500$, and 1000. The finite-size effects appear at increasing times for increasing sizes as expected. A dependence of the slope of the fitted lines with $L$ can be appreciated. This effect is most clearly seen in Fig. 5, which shows the exponents of Table I as a function of $L^{-1}$ (different symbols correspond to different vacancy con-

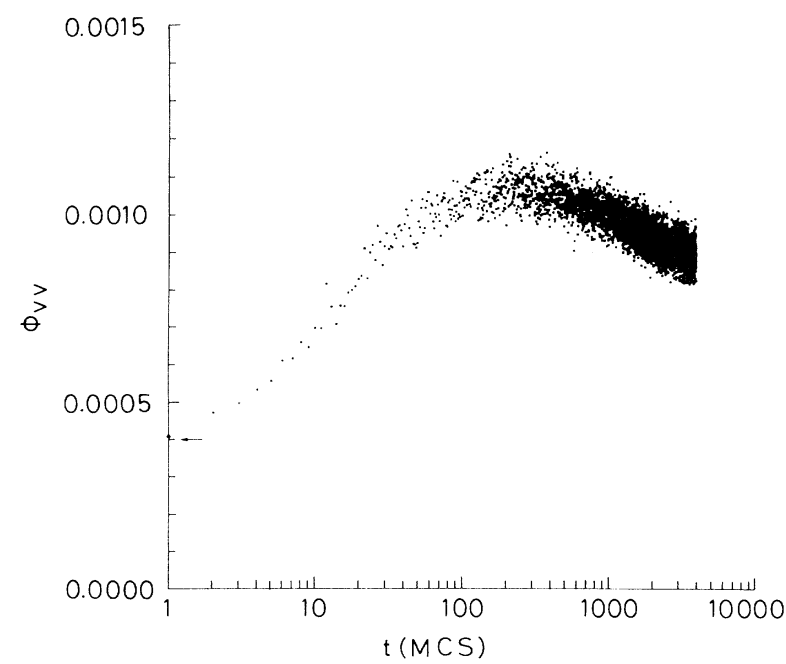

FIG. 3. Number of vacancy-vacancy exchanges per site $\Phi_{V V}$ as a function of time. The arrow indicates the value corresponding to the case in which there is no correlation between vacancies. Data correspond to a system with $L=$ 1000 and $N_{V}=400$ averaged over 13 independent runs. centrations). There is a clear tendency for the exponent to decrease for increasing system sizes. Such a strong dependence with the system size is never seen in the case of the standard Kawasaki exchange mechanism. All the fitted exponents are clearly above the Allen-Cahn value $x=0.5$. Moreover, the results for the $L=1000$ case give an exponent slightly higher (within the data scattering) than the one corresponding to $L=500$. This seems to indicate that the limiting value may also be larger than 0.5 , although the Allen-Cahn value cannot be excluded from these data. Nevertheless, theoretical bounds to this $L \rightarrow \infty$ value can be obtained from consideration of the vacancy diffusion mechanism. ${ }^{21}$ If a vacancy performs a random walk (homogeneous and without memory), its mean-square displacement $\sqrt{\left\langle\mathbf{r}^{2}\right\rangle}$ increases as $t^{y}$ with $y=0.5$ in any dimension. Causality implies that this value gives an upper limit to the growth exponent $x_{\max }=0.5$. Nevertheless, since the vacancy path is correlated with ordering, it cannot be considered to be completely random. In the extreme case, in which the vacancy would perform a self-avoiding walk (it never re-

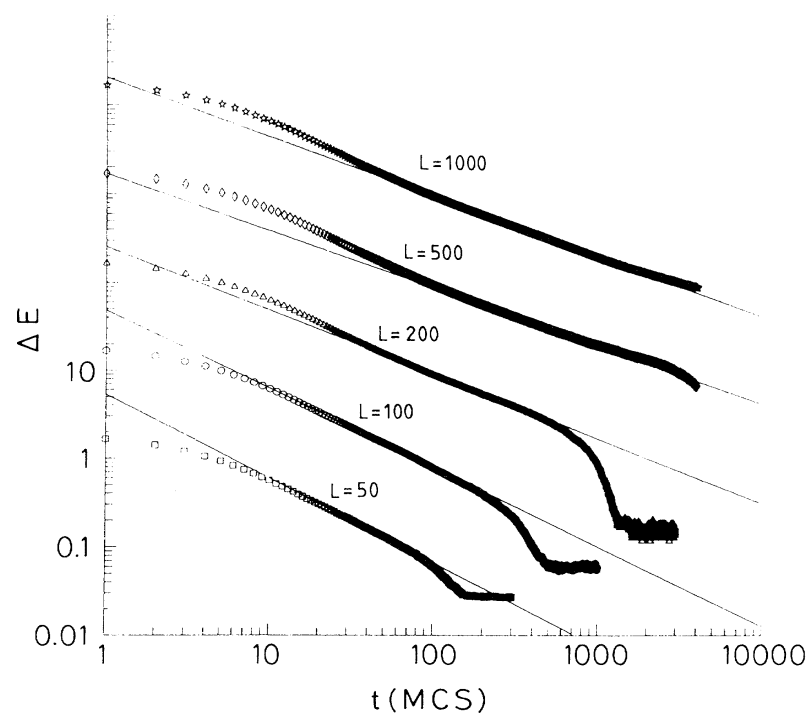

FIG. 4. Log-log plots of the evolution of the excess energy $\Delta E$ versus time for systems of different sizes $L=50,100$, $200,500,1000$, and $c_{V}=4 \times 10^{-4}$. The curves are shifted one decade in order to clarify the picture. The lines are linear square fits whose slope increases for decreasing sizes. 


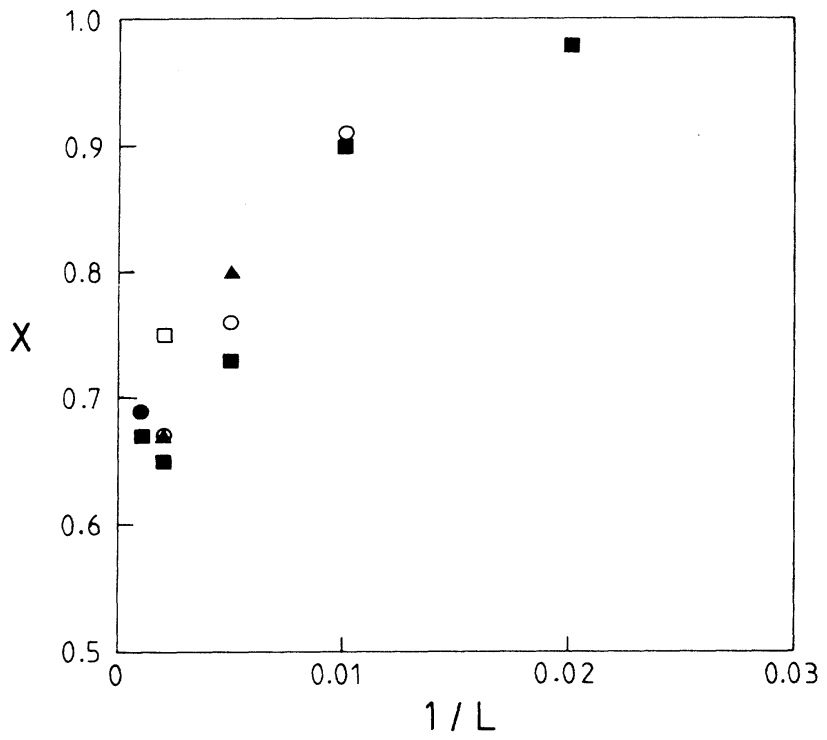

FIG. 5. Fitted growth exponents for different system sizes and vacancy concentrations, as a function of $L^{-1}$. The different symbols correspond to $c_{V}=4 \times 10^{-4}(\square), c_{V}=10^{-4}$ (०), $c_{V}=2.5 \times 10^{-5}(\Delta), c_{V}=4 \times 10^{-6}(\square)$, and $c_{V}=10^{-6}(\bullet)$.

turns to points visited before), $\sqrt{\left\langle\mathbf{r}^{2}\right\rangle} \sim t^{y}$ with $y=0.75$ in a $2 d$ system, giving an upper bound $x_{\max }=0.75$. Actually, the diffusion of the vacancy is a mixture of both processes. Its self-avoiding character comes from the fact that the vacancies prefer to stay in the disordered regions. As a consequence they have little tendency to return to

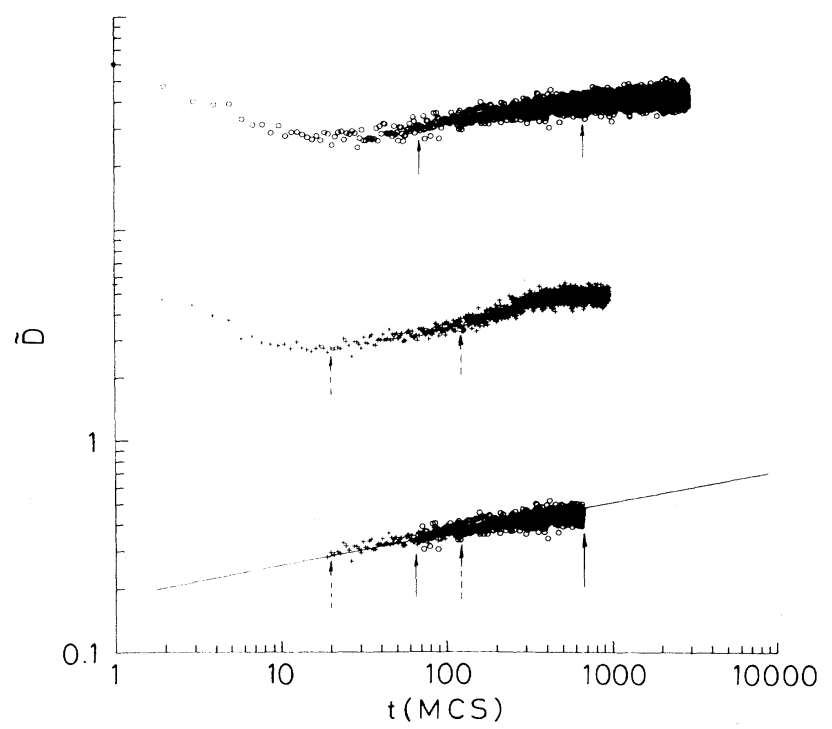

FIG. 6. Log-log plot of the effective diffusion constant $\tilde{D}$ versus time for systems of $L=100(+)$ and $L=500(\circ)$ with a single vacancy. Data correspond to an average over 400 and 200 independent runs, respectively. The bottom curve shows the linear dependence in the domain growth regions, which are indicated by arrows. The straight line is a linear fit to both sets of data simultaneously. The upper curves are shifted one decade for each one. the places that have been visited, since such places are already ordered. This means that in the ordered regions the walks are completely random and less frequent than in the interfaces, where they are quite self-avoiding. Using a generalized scaling hypothesis, one can admit that $\sqrt{\left\langle\mathbf{r}^{2}\right\rangle} \sim R$, which implies that $x=y$. This is equivalent to saying that the growth exponent reaches its causality upper bound value when scaling holds.

Figure 6 shows the effective diffusion constant $\tilde{D}$ defined in Eq. (2), as a function of time, for systems of sizes $L=500$ and 100 with a single vacancy. Arrows indicate the domain growth regime, where the excess energy shows a power-law dependence with time. It can be seen that $\tilde{D}$ is not constant, which indicates that the walk is not a random one. Fitting a power-law dependence $\tilde{D} \sim t^{\alpha}$ renders $\alpha \simeq 0.15$. This value seems to be quite independent of the system size $L$. This result implies that $\sqrt{\left\langle\mathbf{r}^{2}\right\rangle} \sim t^{y}$ with $y=(\alpha+1) / 2 \simeq 0.6$. Taking into account the above scaling argument, this gives rise to a growth exponent $x \simeq 0.6$, which is in reasonable agreement with the tendency of the data in Fig. 5 .

\section{DISCUSSION AND CONCLUSIONS}

The results presented in the preceding section reveal some special features of domain growth in alloys undergoing an order-disorder transition when atomic elementary movements proceed through a vacancy mechanism. First, during the ordering process the vacancy shows an anomalous diffusion, which can be attributed to the nonhomogeneous path of the vacancies arising from its correlation with local order. ${ }^{15}$ This is mainly due to the fact that at low temperatures, when the entropic contribution is negligible, it is favorable for the system to locate the vacancies in disordered regions (domain boundaries). Because NNN jumps are permitted, vacancies follow a random walk in the ordered regions, while the path has some self-avoiding character in the disordered ones. The fast growth exponent $x$ obtained seems to be closely related to this anomalous character of the vacancy diffusion.

Second, when the system contains more than one vacancy, and their concentration is small, our results show that each vacancy behaves independently of the rest. That means that vacancy-vacancy correlations are small enough to have no influence on the growth process. This explains why the domain growth exponent shows very little dependence on the vacancy concentration.

Third, the growth exponent has been found to be strongly influenced by finite-size effects. This dependence of $x$ on $L$, which is never seen when the dynamics is introduced via the standard Kawasaki exchange mechanism, may be associated with the effect of the periodic boundary conditions on the vacancy walk. Nevertheless our results indicate that, even in the $L \rightarrow \infty$ limit (for a given concentration of vacancies), $x$ is greater than 0.5 .

We expect that any change in the system, which makes the path of vacancies more homogeneous, will reduce the growth exponent towards the Allen-Cahn value $x=0.5$. This will happen at high temperature when entropic effects play a relevant role. In other words, high enough temperatures are expected to reduce the self-avoiding 


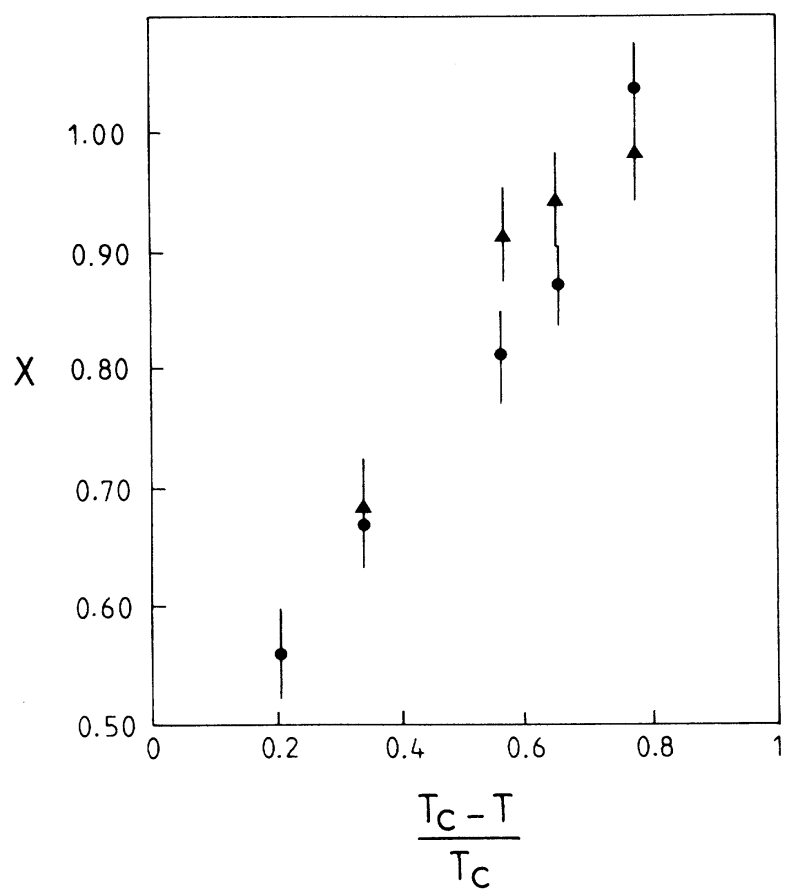

FIG. 7. Growth exponents as a function of the reduced temperature for systems of sizes $L=100(\Delta)$ and $L=200$ $(\bullet)$ with a single vacancy.

character of the vacancy path in the disordered regions. We have checked this by analyzing the temperature dependence of the growth exponent $x$. Figure 7 shows the fitted exponent for systems of $L=100$ and 200 as a function of temperature. The results clearly show that when
$T$ approaches $T_{c}, x$ tends to the Allen-Cahn value. Simulations have not been performed at temperatures too close to the critical temperature in order to avoid the effects coming from the critical slowing down phenomenon. We have also checked the tendency for the exponent $\alpha$ to decrease when the temperature is increased.

This dependence of the exponent $x$ with temperature may explain why exponents larger than $x=0.5$ have not, to our knowledge, been observed experimentally. We believe that this is due to the fact that experimental measurements are always carried out at temperatures quite close to the critical temperature. See, for example, Refs. $6,7,9$, and 12 , where results correspond to temperatures ranging around $T / T_{c} \simeq 0.9$. Experiments at lower temperatures should then be performed in order to test our results.

In relation to the existing theoretical approaches to the domain growth problem, most of them have been formulated assuming that the elementary dynamic excitations are homogeneous and not correlated with the local properties of the system. It might be that some systems exhibit such a homogeneous relaxing behavior after an initial regime, but others may not show it until equilibrium is reached. For low enough temperature, the vacancies in our model for a binary alloy do not behave in such a homogeneous way, even at long times, so the usual theories studying domain growth may not be applicable.

\section{ACKNOWLEDGMENTS}

We are indebted to T. Castán and A. M. Nemirovsky for fruitful discussions. We also acknowledge the Centre de Supercomputació de Catalunya (CESCA) for computing support and CICyT (Spain) for financial support (Project No. MAT92-884).
${ }^{1}$ J.D. Gunton, M. San Miguel, and P.S. Sahni, in Phase Transitions and Critical Phenomena, edited by C. Domb and J.L. Lebowitz (Academic, London, 1983), Vol. 8.

${ }^{2}$ O.G. Mouritsen, in Kinetics of Ordering and Growth at Surfaces, edited by M.G. Lagally (Plenum, New York, 1990).

${ }^{3}$ G.F. Mazenko, Phys. Rev. B 43, 8204 (1991).

${ }^{4}$ A. Sadiq and K. Binder, J. Stat. Phys. 35, 517 (1984).

${ }^{5}$ O.T. Valls and G.F. Mazenko, Phys. Rev. B 34, 7941 (1986).

${ }^{6}$ R.F. Shannon, S.E. Nagler, C.R. Harkless, and R.M. Nicklow, Phys. Rev. B 46, 40 (1992).

${ }^{7}$ S.E. Nagler, R.F. Shannon, Jr., C.R. Harkless, and M.A. Sing, Phys. Rev. Lett. 61, 718 (1988).

${ }^{8}$ J.-K. Zuo, G.-C. Wang, and T.-M. Lu, Phys. Rev. Lett. 60, 1053 (1988).

${ }^{9}$ S. Katano, M. Iizumi, R.M. Nicklow, and H.R. Child, Phys. Rev. B, 38, 2659 (1988).

${ }^{10}$ M.K. Phani, J.L. Lebowitz, M.H. Kalos, and O. Penrose, Phys. Rev. Lett. 45, 366 (1980).

${ }^{11}$ P.S. Sahni, G. Dee, J.D. Gunton, M. Phani, and J.L. Lebowitz, Phys. Rev. B, 24, 410 (1981).
${ }^{12}$ S.M. Allen and J.W. Cahn, Acta Metall. 27, 1085 (1979).

${ }^{13}$ See, for instance, J. Philibert, Diffusion et Transport de Matière dans les Solides (Editions de Physique, Orsay, 1985), and references therein.

${ }^{14}$ E. Vives and A. Planes, Phys. Rev. Lett. 68, 812 (1992).

${ }^{15}$ E. Vives and A. Planes, Phys. Rev. B 47, 2557 (1993).

${ }^{16}$ O.G. Mouritsen and P.J. Shah, Phys. Rev. B 40, 11445 (1989).

${ }^{17}$ P.J. Shah and O.G. Mouritsen, Phys. Rev. B 41, 7003 (1990).

${ }^{18}$ The $A B V$ model has been used for the study of the phase separation problem in binary alloys. See K. Yaldram and K. Binder, Z. Phys. B 82, 405 (1991); J. Stat. Phys. 62, 161 (1991); Acta Metall. Mater. 39, 707 (1991).

${ }^{19}$ E. Vives and A. Planes, Int. J. Mod. Phys. C (to be published).

${ }^{20}$ K. Binder, J. Comp. Phys. 59, 1 (1988).

${ }^{21} \mathrm{An}$ introduction to random walkers and diffusion can be found in E.P. Raposo, S.M. de Oliveira, A.M. Nemirovsky, and M.D. Coutinho-Filho, Am. J. Phys. 59, 633 (1991). 